\title{
Spatially resolved coherent 2D fluorescence spectroscopy within a high-NA microscope
}

\author{
Donghai $\mathrm{Li}^{1,{ }^{*}}$, Matthias $\mathrm{Nuss}^{1}$, Sebastian Goetz ${ }^{1}$, Verena $\mathrm{Kolb}^{2}$, Jens Pflaum ${ }^{2,3}$, Chiara \\ Trovatello $^{4}$, Giulio Cerullo ${ }^{4,5}$, and Tobias Brixner ${ }^{1,6}$ \\ ${ }^{1}$ Institut für Physikalische und Theoretische Chemie, Universität Würzburg, Am Hubland, 97074 \\ Würzburg, Germany \\ ${ }^{2}$ Experimental Physics VI, Universität Würzburg, Am Hubland, 97074 Würzburg, Germany \\ ${ }^{3}$ Bavarian Center for Applied Energy Research, Magdalene-Schoch-Str. 3, 97074 Würzburg, \\ Germany \\ ${ }^{4}$ Dipartimento di Fisica, Politecnico di Milano, Piazza Leonardo da Vinci 32, 20133 Milano, Italy \\ ${ }^{5}$ Istituto di Fotonica e Nanotecnologie, CNR, Piazza Leonardo da Vinci 32, 20133 Milano, Italy \\ ${ }^{6}$ Center for Nanosystems Chemistry, Theodor-Boveri-Weg, 97074 Würzburg, Germany
}

\begin{abstract}
We have developed coherent two-dimensional (2D) fluorescence micro-spectroscopy which probes the nonlinear optical response at surfaces via fluorescence detection with sub-micron spatial resolution. This enables the investigation of microscopic variations in laterally heterogeneous film samples which are of interests for subwavelength opto-electronic devices.
\end{abstract}

\section{Introduction}

Coherent two-dimensional (2D) spectroscopy reveals the third-order nonlinear response of the system under investigation. Most geometries detect a coherently emitted four-wavemixing signal following three-pulse excitation. However, when a fully collinear geometry is chosen $[1,2]$, incoherent population-based observables can also be utilized as has been demonstrated for fluorescence [1-3], mass spectroscopy [4], or electron currents [5]. Collinear geometry offers a possibility to couple the laser pulses into a high-NA fluorescence microscope. With such a method, a spatial resolution below half of the wavelength of the exciting electromagnetic field can be achieved according to the diffraction limit. In this work, we establish spatially resolved coherent $2 \mathrm{D}$ fluorescence microscopy and present two examples to demonstrate its applications.

\section{Experimental methods}

The experimental setup is described in detail in a previous publication [6]. Basically, a femtosecond oscillator is used to provide broadband fs laser pulses with a spectrum ranging from $670 \mathrm{~nm}$ to $810 \mathrm{~nm}$. A pulse shaper is used to generate pulse sequences with

\footnotetext{
* Corresponding author: donghai.li@phys-chemie.uni-wuerzburg.de
} 
controllable inter-pulse time delays and phases for the linear and 2D spectral measurements. The laser focus in the microscope is mapped by a piezo scanning stage. Sample fluorescence is detected by an avalanche photodiode (APD).

Linear spectra as well as nonlinear 2D spectra can be obtained at different positions on the sample surface with spatial resolution of $260 \mathrm{~nm}$. For linear spectra, a two-pulse delay scan is conducted, where fluorescence intensity is recorded as a function of time delay between the two pulses. Subsequent Fourier transformation results in a spectrum which is the product of the laser spectrum and the fluorescence excitation spectrum. So, by dividing by the laser spectrum, the spatially resolved excitation spectrum can be obtained. While in the 2D spectral measurements, relative time delays between four pulses are scanned, namely coherence time $\tau$, the population time $T$, and signal time $t$. A 27-step phase-cycling scheme is used, allowing the extraction of the absorptive spectrum from the rephasing and non-rephasing nonlinear contributions.

\section{Results and discussions}

As the first sample, a regularly structured sample surface made of fluorinated zinc phthalocyanine $\left(\mathrm{F}_{16} \mathrm{ZnPc}\right)$ is fabricated, the process is detailed in reference [6]. The absorption spectrum of a planar $\mathrm{F}_{16} \mathrm{ZnPc}$ film is fitted by multiple peaks as shown in Fig. 1(a) and indicates the absorption features that result from different coexisting molecular conformations upon preparation. By measuring linear spectra from an area of interest, a gradient spectral change across the molecular "hills" can be seen in Fig. 1(b). Typical linear spectra are shown in Figs. 1(c) and 1(d) for two exemplary locations. The two excitation spectra show differences in the ratio between intensities of high- and low-frequency components. 2D absorptive spectra are measured at the same positions and are shown below the corresponding linear spectra [Figs. 1(e) and 1(f)]. Along the diagonal, the observed difference in the ratio between the two components is similar to the linear spectra. Broadening in the anti-diagonal direction in the two 2D spectra indicates strong coupling between the high- and low-frequency components.
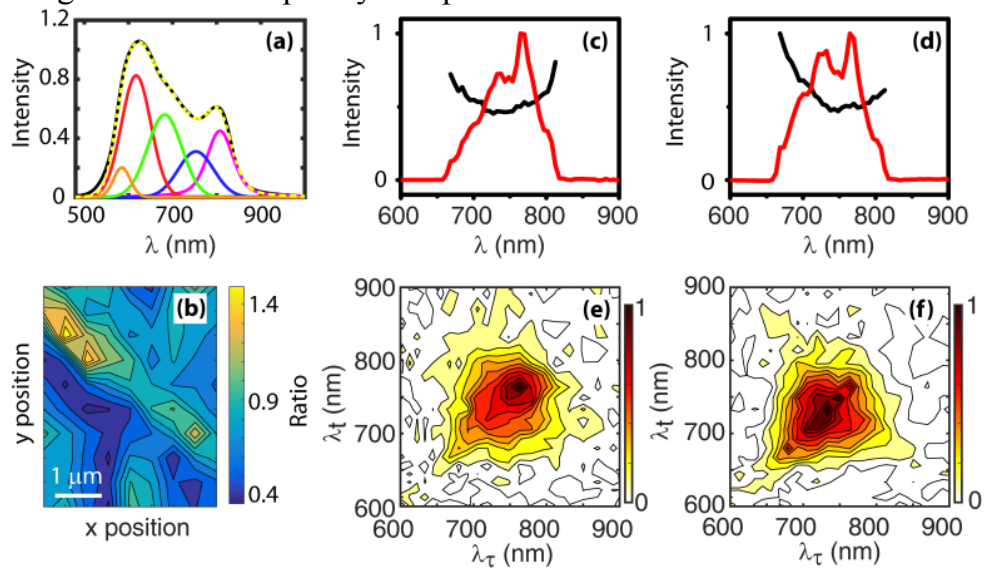

Fig. 1. (a) Absorption spectrum (black solid line) of a planar $\mathrm{F}_{16} \mathrm{ZnPc}$ film, fitting curve (yellow dotted line) with multiple component peaks plotted in different colors. (b) Map of ratio between intensities at 700 and $770 \mathrm{~nm}$ in linear spectra. (c, d) Spatially resolved linear product spectra (red) and excitation spectra (black) from two exemplary positions of the sample. (e, f) Corresponding spatially resolved $2 \mathrm{D}$ absorptive spectra from the two exemplary positions.

As a second sample, a small area of monolayer region on a MoSe 2 surface (about $10 \mu \mathrm{m}$ in diameter) is studied. The absorption spectrum of the $\mathrm{MoSe}_{2}$ monolayer has two peaks 
corresponding to transition bands labeled A and B [7]. 2D fluorescence micro-spectra at different waiting times were measured as shown in Fig. 2(a). A strong diagonal peak of transition band $\mathrm{A}$ and two cross peaks corresponding to energy transfer from $\mathrm{A}$ to $\mathrm{B}$ and from B to A can be clearly seen. The cross-peak dynamics are extracted as the curves in Fig. 2(b). As waiting time increases, the B-to-A transfer peak rises. The fine structure of peak A changes with waiting time dramatically. Plotting the evolution of different regions within diagonal peak A as marked by green and purple dashed lines in Fig. 2(a), long-lived out-ofphase oscillations lasting up to 2 ps can be observed as shown in Fig. 2(c).
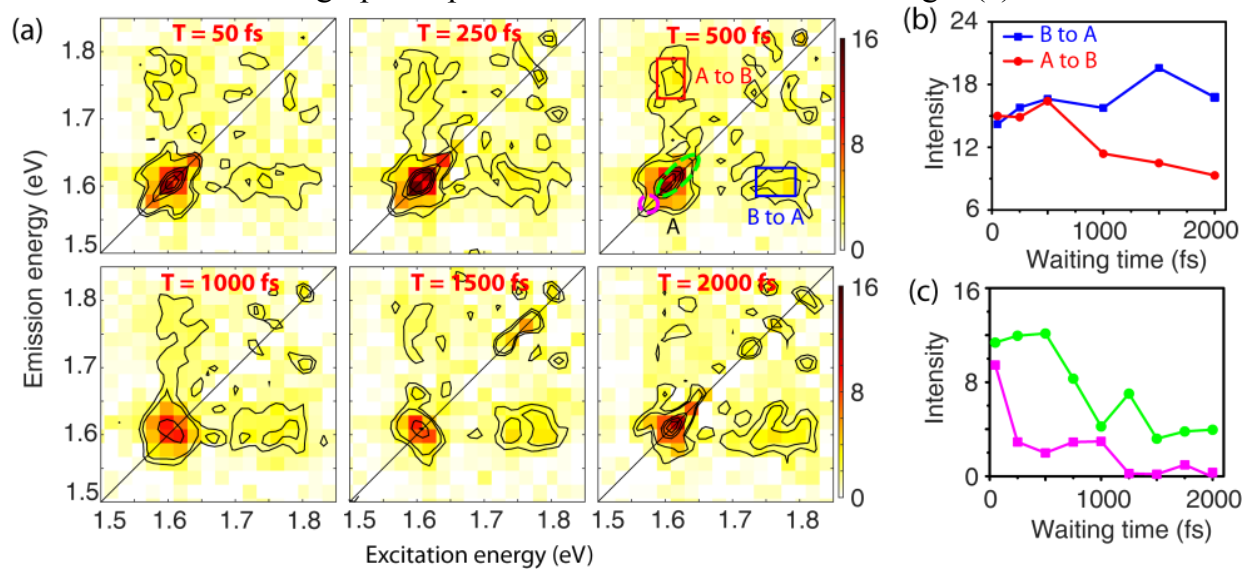

Fig. 2. (a) $2 \mathrm{D}$ absorptive spectra at different waiting times. In the map for $T=500 \mathrm{fs}$, four regions of interest are marked with different colors. (b) Averaged intensity in the marked cross-peak areas as a function of waiting time (red: transfer from A to B, blue: transfer from B to A). (c) Averaged intensity in the two marked regions (green and purple) within peak A as a function of waiting time.

\section{Conclusions}

In conclusion, we established the method of spatially resolved 2D fluorescence microscopy, where high temporal and spatial resolutions are achieved at the same time. Two samples were studied. The first one shows that $2 \mathrm{D}$ spectra can be measured from distinct small domains, and localized structural information can be extracted. The second study shows that ultrafast energy transfer dynamics within a microscopic target can be well detected by this method.

\section{References}

1. P. Tian, D. Keusters, Y. Suzaki, and W. S. Warren, Science 300, 1553 (2003).

2. P. F. Tekavec, G. A. Lott, and A. H. Marcus, J. Chem. Phys. 127, 214307 (2007).

3. S. Draeger, S. Roeding, and T. Brixner, Opt. Express 25, 3259 (2017).

4. S. Roeding and T. Brixner, Nat. Commun. 9, 259 (2018).

5. M. Aeschlimann, T. Brixner, A. Fischer, C. Kramer, P. Melchior, W. Pfeiffer, C. Schneider, C. Strüber, P. Tuchscherer, and D. V. Voronine, Science 333, 1723 (2011).

6. S. Goetz, D. Li, V. Kolb, J. Pflaum, and T. Brixner, Opt. Express 26, 3915 (2018).

7. Z. Nie, C. Trovatello, E. A. A. Pogna, S. Dal Conte, P. B. Miranda, E. Kelleher, C. Zhu, I. C. E. Turcu, Y. Xu, K. Liu, G. Cerullo, and F. Wang, Appl. Phys. Lett. 112, 031108 (2018) 